\title{
Local multiplicity fluctuations in hadronic Z decay
}

\author{
L3 Collaboration
}

M. Acciarri ${ }^{\text {aa }}$, O. Adriani ${ }^{\text {p }}$, M. Aguilar-Benitez ${ }^{\text {z }}$, S. Ahlen ${ }^{\text {k }}$, J. Alcaraz ${ }^{\mathrm{z}}$,

G. Alemanni v , J. Allaby ${ }^{q}$, A. Aloisio ${ }^{\text {ac }}$, M.G. Alviggi ${ }^{\text {ac }}$, G. Ambrosi ${ }^{\text {s, }}$, H. Anderhub aw, V.P. Andreev al, T. Angelescu ${ }^{\mathrm{m}}$, F. Anselmo i, A. Arefiev ${ }^{\text {ab }}$, T. Azemoon ${ }^{\text {c }}$, T. Aziz ${ }^{j}$, P. Bagnaia ${ }^{\text {ak }}$, L. Baksay ${ }^{\text {ar }}$, R.C. Ball ${ }^{\text {c }}$, S. Banerjee ${ }^{j}$, Sw. Banerjee ${ }^{\mathrm{j}}, \mathrm{K}$. Banicz ${ }^{\text {at }}$, A. Barczyk ${ }^{\text {aw,au }}$, R. Barillère ${ }^{\mathrm{q}}$, L. Barone ${ }^{\text {ak }}$, P. Bartalini ah ${ }^{\text {ah }}$ A. Baschirotto ${ }^{\text {aa }}$, M. Basile ${ }^{\mathrm{i}}$, R. Battiston ${ }^{\text {ah }}$, A. Bay ${ }^{\mathrm{v}}$, F. Becattini ${ }^{\mathrm{p}}$, U. Becker ${ }^{\circ}$, F. Behner ${ }^{\text {aw }}$, J. Berdugo ${ }^{\text {z }}$, P. Berges ${ }^{\circ}$, B. Bertucci ${ }^{\text {ah }}$, B.L. Betev ${ }^{\text {aw }}$, S. Bhattacharya ${ }^{j}$, M. Biasini ${ }^{\mathrm{q}}$, A. Biland ${ }^{\text {aw }}$, G.M. Bilei ah , J.J. Blaising ${ }^{\mathrm{d}}$, S.C. Blyth ${ }^{\text {ai }}$, G.J. Bobbink ${ }^{\text {b }}$, R. Bock ${ }^{\text {a }}$, A. Böhm ${ }^{\text {a }}$, L. Boldizsar ${ }^{\text {n }}$, B. Borgia ${ }^{\text {ak }}$,

D. Bourilkov ${ }^{\text {aw }}$, M. Bourquin s, D. Boutigny ", S. Braccini s, J.G. Branson an, V. Brigljevic ${ }^{\text {aw }}$, I.C. Brock ${ }^{\text {ai }}$, A. Buffini ${ }^{p}$, A. Buijs ${ }^{\text {as }}$, J.D. Burger ${ }^{\circ}$, W.J. Burger ${ }^{\mathrm{s}}$, J. Busenitz ${ }^{\text {ar }}$, X.D. Cai ${ }^{\circ}$, M. Campanelli ${ }^{\text {aw }}$, M. Capell ${ }^{\circ}$, G. Cara Romeo i,

G. Carlino ${ }^{\text {ac }}$, A.M. Cartacci ${ }^{\mathrm{p}}$, J. Casaus ${ }^{\mathrm{z}}$, G. Castellini ${ }^{\mathrm{p}}$, F. Cavallari ${ }^{\text {ak }}$, N. Cavallo ac, C. Cecchi s, M. Cerrada ${ }^{\text {z }}$, F. Cesaroni ${ }^{\text {w }}$, M. Chamizo ${ }^{\text {z }}$, Y.H. Chang ay, U.K. Chaturvedi ', S.V. Chekanov ${ }^{\text {ae }}$, M. Chemarin ${ }^{y}$, A. Chen ${ }^{\text {ay }}$, G. Chen ${ }^{\mathrm{g}}$, G.M. Chen ${ }^{\mathrm{g}}$, H.F. Chen ${ }^{\mathrm{t}}$, H.S. Chen ${ }^{\mathrm{g}}$, M. Chen ${ }^{\circ}$, G. Chiefari ${ }^{\mathrm{ac}}$, C.Y. Chien ${ }^{\mathrm{e}}$, L. Cifarelli ${ }^{\mathrm{am}}$, F. Cindolo $^{\mathrm{i}}$, C. Civinini ${ }^{\mathrm{p}}$, I. Clare ${ }^{\circ}$, R. Clare ${ }^{o}$, H.O. Cohn ${ }^{\text {af }}$, G. Coignet ${ }^{\text {d }}$, A.P. Colijn ${ }^{\text {b }}$, N. Colino ${ }^{\mathrm{z}}$, S. Costantini ${ }^{\text {h }}$, F. Cotorobai ${ }^{m}$, B. de la Cruz ${ }^{z}$, A. Csilling ${ }^{n}$, T.S. Dai ${ }^{\circ}$, R. D'Alessandro ${ }^{p}$, R. de Asmundis ${ }^{\text {ac }}$, A. Degré ${ }^{d}$, K. Deiters au, P. Denes ${ }^{\text {aj }}$, F. DeNotaristefani ${ }^{\text {ak }}$, D. DiBitonto ${ }^{\text {ar }}, \mathrm{M}$. Diemoz ${ }^{\text {ak }}$, D. van Dierendonck ${ }^{\text {b }}$, F. Di Lodovico ${ }^{\text {aw }}$, C. Dionisi ${ }^{\text {ak }}$, M. Dittmar aw ${ }^{\text {a }}$ A. Dominguez ${ }^{\text {an }}$, A. Doria ${ }^{\text {ac }}$, M.T. Dova ${ }^{\mathrm{r}, 1}$, E. Drago ${ }^{\text {ac }}$, D. Duchesneau d, P. Duinker ${ }^{\text {b }}$, I. Duran ${ }^{\text {ao }}$, S. Dutta ${ }^{\text {j }}$, S. Easo ${ }^{\text {ah }}$, Yu. Efremenko ${ }^{\text {af }}$, H. El Mamouni ${ }^{y}$, A. Engler ${ }^{\text {ai }}$, F.J. Eppling ${ }^{\circ}$, F.C. Erné ${ }^{b}$, J.P. Ernenwein ${ }^{y}$, P. Extermann ${ }^{\text {s }}$, M. Fabre ${ }^{\text {au }}$, R. Faccini ${ }^{\text {ak }}$, S. Falciano ${ }^{\text {ak }}$, A. Favara ${ }^{\mathrm{p}}$, J. Fay ${ }^{\mathrm{y}}$, O. Fedin ${ }^{\text {al }}$, M. Felcini ${ }^{\mathrm{aw}}$, B. Fenyi ${ }^{\text {ar }}$, T. Ferguson ${ }^{\text {ai }}$, F. Ferroni ${ }^{\text {ak }}$, H. Fesefeldt ${ }^{\text {a }}$, E. Fiandrini ${ }^{\text {ah }}$, J.H. Field ${ }^{\text {s }, ~ F . ~ F i l t h a u t ~}{ }^{\text {ai }}$, P.H. Fisher ${ }^{0}$, I. Fisk $^{\text {an }}$, G. Forconi ${ }^{\circ}$, L. Fredj ${ }^{\text {s, K. Freudenreich }}{ }^{\text {aw }}$, C. Furetta ${ }^{\text {aa }}$, 
Yu. Galaktionov ${ }^{\text {ab,o }}$, S.N. Ganguli ${ }^{\text {j, }}$ P. Garcia-Abia ${ }^{\mathrm{f}}$, S.S. Gau ${ }^{\text {I }}$, S. Gentile ${ }^{\mathrm{ak}}$, J. Gerald $^{\mathrm{e}}$, N. Gheordanescu ${ }^{\mathrm{m}}$, S. Giagu ${ }^{\mathrm{ak}}$, S. Goldfarb ${ }^{\mathrm{v}}$, J. Goldstein ${ }^{\mathrm{k}}$, Z.F. Gong ${ }^{\mathrm{t}}$, A. Gougas ${ }^{\mathrm{e}}$, G. Gratta ${ }^{\text {ag }}$, M.W. Gruenewald ${ }^{\text {h }}$, V.K. Gupta ${ }^{\text {aj }}$, A. Gurtu ${ }^{\text {j, L.J. Gutay }}{ }^{\text {at }}$, D. Haas ${ }^{\text {f }}$, B. Hartmann ${ }^{\text {a }}$, A. Hasan ${ }^{\text {ad }}$, D. Hatzifotiadou ${ }^{\text {i }}$, T. Hebbeker ${ }^{\text {h }}$, A. Hervé ${ }^{\text {q }}$ J. Hirschfelder ai, W.C. van Hoek ${ }^{\text {ae }}$, H. Hofer ${ }^{\text {aw }}$, S.J. Hong aq, H. Hoorani ${ }^{\text {ai }}$, S.R. Hou ${ }^{\text {ay }}$, G. Hu ${ }^{\text {e }}$, V. Innocente ${ }^{\mathrm{q}}$, K. Jenkes ${ }^{\text {a }}$, B.N. Jin ${ }^{g}$, L.W. Jones ${ }^{c}$, P. de Jong ${ }^{q}$, I. Josa-Mutuberria ${ }^{z}$, A. Kasser ${ }^{\mathrm{v}}$, R.A. Khan ${ }^{r}$,D. Kamrad ${ }^{\text {av }}$, Yu. Kamyshkov af , J.S. Kapustinsky ${ }^{x}$, Y. Karyotakis ${ }^{\text {d }}$, M. Kaur ${ }^{\mathrm{r}, 2}$,M.N. Kienzle-Focacci ${ }^{\text {s }}$, D. Kim ${ }^{\text {ak }}$, D.H. Kim ${ }^{\text {aq }}$, J.K. Kim ${ }^{\text {aq }}$, S.C. Kim ${ }^{\text {aq }}$, Y.G. Kim ${ }^{\text {aq }}$, W.W. Kinnison ${ }^{x}$, A. Kirkby ${ }^{\text {ag }}$, D. Kirkby ${ }^{\text {ag }}$, J. Kirkby ${ }^{\text {q }}$, D. Kiss ${ }^{\text {n, W. Kittel }}{ }^{\text {ae }}$, A. Klimentov ${ }^{\text {o,ab }}$, A.C. König ${ }^{\text {ae }}$, A. Kopp ${ }^{\text {av }}$, I. Korolko ${ }^{\text {ab }}$, V. Koutsenko ${ }^{\text {o,ab }}$, R.W. Kraemer ${ }^{\text {ai }}$, W. Krenz ${ }^{a}$, A. Kunin ${ }^{o, a b}$, P. Lacentre ${ }^{a v, 1},{ }^{3}$

P. Ladron de Guevara ${ }^{z}$, G. Landi ${ }^{p}$, C. Lapoint ${ }^{\circ}$, K. Lassila-Perini ${ }^{\text {aw }}$, P. Laurikainen " ${ }^{\text {, A. Lavorato }}{ }^{\mathrm{am}}, \mathrm{M}$. Lebeau ${ }^{\mathrm{q}}$, A. Lebedev ${ }^{\mathrm{o}}$, P. Lebrun ${ }^{\mathrm{y}}$, P. Lecomte ${ }^{\text {aw }}$,P. Lecoq ${ }^{q}$, P. Le Coultre ${ }^{\text {aw }}$, H.J. Lee ${ }^{\text {h}}$, C. Leggett ${ }^{c}$, J.M. Le Goff ${ }^{q}$, R. Leiste ${ }^{\text {av }}$, E. Leonardi ak ${ }^{\text {a }}$ P. Levtchenko ${ }^{\text {al }}$, C. Li ${ }^{\text {t }}$, C.H. Lin ${ }^{\text {ay }}$, W.T. Lin ${ }^{\text {ay }}$, F.L. Linde ${ }^{\mathrm{b}, \mathrm{q}}$, L. Lista $^{\mathrm{ac}}$, Z.A. Liu ${ }^{\mathrm{g}}$, W. Lohmann ${ }^{\text {av }}$, E. Longo ${ }^{\text {ak }}$, W. Lu ${ }^{\mathrm{ag}}$, Y.S. Lu ${ }^{\mathrm{g}}, \mathrm{K}$. Lübelsmeyer ${ }^{\text {a }}$, C. Luci ${ }^{\text {ak }}$, D. Luckey ${ }^{\circ}$, L. Luminari ${ }^{\text {ak }}$, W. Lustermann ${ }^{\text {au }}$, W.G. Ma ${ }^{\mathrm{t}}$, M. Maity ${ }^{\mathrm{j}}$, G. Majumder ${ }^{\mathrm{j}}$, L. Malgeri ${ }^{\text {ak }}$, A. Malinin ${ }^{\text {ab }}$, C. Maña ${ }^{\text {z }}$, D. Mangeol ${ }^{\text {ae }}$, S. Mangla ${ }^{\text {j, P. Marchesini }}{ }^{\text {aw }}$, A. Marin ${ }^{\text {, }}$, J.P. Martin ${ }^{\mathrm{y}}$, F. Marzano ${ }^{\mathrm{ak}}$, G.G.G. Massaro ${ }^{\mathrm{b}}$, D. McNally ${ }^{\mathrm{q}}$, S. Mele ${ }^{\mathrm{ac}}$, L. Merola $^{\text {ac }}$, M. Meschini ${ }^{\text {p }}$, W.J. Metzger ${ }^{\text {ae }}$, M. von der Mey ${ }^{\text {a }}$, Y. Mi v, D. Migani ${ }^{\mathrm{i}}$, A. Mihul ${ }^{\mathrm{m}}$, A.J.W. van Mil ${ }^{\text {ae }}$, H. Milcent ${ }^{\mathrm{q}}$, G. Mirabelli ${ }^{\text {ak }}$, J. Mnich ${ }^{\mathrm{q}}$, P. Molnar ${ }^{\mathrm{h}}$, B. Monteleoni ${ }^{\mathrm{p}}$, R. Moore ${ }^{\mathrm{c}}$, S. Morganti ${ }^{\text {ak }}$, T. Moulik ${ }^{\mathrm{j}}$, R. Mount ${ }^{\mathrm{ag}}$, F. Muheim ${ }^{\text {s }}$, A.J.M. Muijs ${ }^{b}$, S. Nahn ${ }^{\circ}$, M. Napolitano ${ }^{\text {ac }}$, F. Nessi-Tedaldi ${ }^{\text {aw }}$,

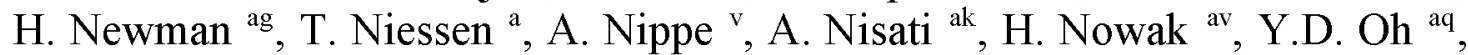
H. Opitz a , G. Organtini ak , R. Ostonen " , S. Palit ${ }^{~}$, C. Palomares ${ }^{z}$, D. Pandoulas a , S. Paoletti ${ }^{\text {ak }}$, P. Paolucci ${ }^{\text {ac }}$, H.K. Park ai ${ }^{\text {a }}$ I.H. Park ${ }^{\text {aq }}$, G. Pascale ${ }^{\text {ak }}$, G. Passaleva ${ }^{q}$, S. Patricelli ac, T. Paul ${ }^{1}$, M. Pauluzzi ${ }^{\text {ah }}$, C. Paus ${ }^{\mathrm{q}}$, F. Pauss ${ }^{\text {aw }}$, D. Peach ${ }^{\mathrm{q}}$, Y.J. Pei a , S. Pensotti ${ }^{\text {aa }}$, D. Perret-Gallix ${ }^{\text {d }}$, B. Petersen ${ }^{\text {ae }}$, S. Petrak ${ }^{\text {h }}$, A. Pevsner ${ }^{e}$, D. Piccolo ${ }^{a c}$, M. Pieri ${ }^{p}$, P.A. Piroué ${ }^{\text {aj }}$, E. Pistolesi ${ }^{\text {aa }}$, V. Plyaskin ${ }^{\text {ab }}$, M. Pohl aw, V. Pojidaev ab,p, H. Postema ${ }^{\circ}$, N. Produit ${ }^{\text {s }}$, D. Prokofiev al, J. Quartieri ${ }^{\text {am }}$, G. Rahal-Callot ${ }^{\text {aw }}$, N. Raja ${ }^{j}$, P.G. Rancoita ${ }^{\text {aa }}$, M. Rattaggi ${ }^{\text {aa }}$, G. Raven ${ }^{\text {an }}$, P. Razis ${ }^{\text {ad }}$, K. Read ${ }^{\text {af }}$, D. Ren ${ }^{\text {aw }}$, M. Rescigno ${ }^{\text {ak }}$, S. Reucroft ${ }^{1}$,T. van Rhee ${ }^{\text {as }}$, S. Riemann ${ }^{\text {av }}$, K. Riles ${ }^{\text {c }}$, O. Rind ${ }^{\text {c }}$, A. Robohm ${ }^{\text {aw }}$, J. Rodin ${ }^{\circ}$, B.P. Roe ${ }^{c}$, L. Romero ${ }^{z}$, S. Rosier-Lees ${ }^{d}$, Ph. Rosselet ${ }^{v}$, W. van Rossum as , S. Roth ${ }^{\text {a }}$, J.A. Rubio ${ }^{\mathrm{q}}$, D. Ruschmeier ${ }^{\mathrm{h}}$, H. Rykaczewski ${ }^{\text {aw }}$, J. Salicio ${ }^{\text {q }}$, E. Sanchez ${ }^{\text {z }}$, M.P. Sanders ${ }^{\text {ae }}$, M.E. Sarakinos ", S. Sarkar ${ }^{\text {j, }}$ 
G. Sauvage ${ }^{\text {d }}$, C. Schäfer ${ }^{\text {a }}$, V. Schegelsky ${ }^{\text {al }}$, S. Schmidt-Kaerst ${ }^{\text {a }}$, D. Schmitz a M. Schneegans ${ }^{\text {d }}$, N. Scholz ${ }^{\text {aw }}$, H. Schopper ${ }^{\text {ax }}$, D.J. Schotanus ${ }^{\text {ae }}$, J. Schwenke ${ }^{\text {a }}$, G. Schwering a, C. Sciacca ${ }^{\text {ac }}$, D. Sciarrino s, L. Servoli ah, S. Shevchenko ${ }^{\text {ag }}$, N. Shivarov ap, V. Shoutko ${ }^{\text {ab }}$, J. Shukla ${ }^{x}$, E. Shumilov ${ }^{\text {ab }}$, A. Shvorob ${ }^{a g}$, T. Siedenburg ${ }^{\text {a }}$, D. Son ${ }^{\text {aq }}$, V. Soulimov ${ }^{\text {ac }}$, B. Smith ${ }^{\circ}$, P. Spillantini ${ }^{\text {p }}$, M. Steuer ${ }^{\circ}$, D.P. Stickland ${ }^{\text {aj }}$, H. Stone ${ }^{\text {aj }}$, B. Stoyanov ${ }^{\text {ap }}$, A. Straessner ${ }^{\text {a }}$, K. Sudhakar ${ }^{\mathrm{j}}$, G. Sultanov ${ }^{r}$, L.Z. Sun ${ }^{\mathrm{t}}$, G.F. Susinno ${ }^{\mathrm{s}}$, H. Suter ${ }^{\text {aw }}$, J.D. Swain ${ }^{\mathrm{r}}$, X.W. Tang ${ }^{\mathrm{g}}$, L. Tauscher ${ }^{f}$, L. Taylor ${ }^{1}$, Samuel C.C. Ting ${ }^{\circ}$, S.M. Ting ${ }^{\circ}$, S.C. Tonwar ${ }^{j}$, J. Tóth ${ }^{\mathrm{n}}$, C. Tully ${ }^{\text {aj }}$, H. Tuchscherer ${ }^{\text {ar }}$, K.L. Tung ${ }^{\mathrm{g}}$, Y. Uchida ${ }^{\mathrm{o}}$, J. Ulbricht ${ }^{\text {aw }}$, U. Uwer ${ }^{\mathrm{q}}$, E. Valente ${ }^{\mathrm{ak}}, \mathrm{G}$. Vesztergombi ${ }^{\mathrm{n}}$, I. Vetlitsky ${ }^{\mathrm{ab}}, \mathrm{G}$. Viertel ${ }^{\mathrm{aw}}$, M. Vivargent ${ }^{\text {d, }}$ S. Vlachos ${ }^{\text {f }}$, R. Völkert ${ }^{\text {av }}$, H. Vogel ${ }^{\text {ai }}$, H. Vogt ${ }^{\text {av }}$, I. Vorobiev ${ }^{\text {qab }}$, A.A. Vorobyov ${ }^{\text {al }}$, A. Vorvolakos ${ }^{\text {ad }}$, M. Wadhwa ${ }^{\text {f }}$, W. Wallraff a , J.C. Wang ${ }^{\circ}$, X.L. Wang ${ }^{\mathrm{t}}$, Z.M. Wang ${ }^{\mathrm{t}}$, A. Weber ${ }^{\mathrm{a}}$, S.X. Wu ${ }^{\mathrm{o}}$, S. Wynhoff ${ }^{\mathrm{a}}$, J. Xu ${ }^{\mathrm{k}}, \mathrm{Z} . Z$. Xu ${ }^{\mathrm{t}}$, B.Z. Yang ${ }^{\mathrm{t}}$, C.G. Yang ${ }^{\mathrm{g}}$, X.Y. Yao ${ }^{\mathrm{g}}$, J.B. Ye ${ }^{\mathrm{t}}$, S.C. Yeh ${ }^{\text {ay }}$, J.M. You ${ }^{\text {ai }}$, An. Zalite ${ }^{\text {al }}$, Yu. Zalite ${ }^{\text {al }}$, P. Zemp ${ }^{\text {aw }}$, Y. Zeng ${ }^{\text {a }}$, Z. Zhang ${ }^{\mathrm{g}}$, Z.P. Zhang ${ }^{\mathrm{t}}$, B. Zhou ${ }^{k}$,Y. Zhou ${ }^{\text {c }}$, G.Y. Zhu ${ }^{\text {g }}$, R. Y. Zhu ${ }^{\text {ag }}$, A. Zichichi ${ }^{\text {i,q,r }}$, F. Ziegler ${ }^{\text {av }}$

${ }^{a}$ I. Physikalisches Institut, RWTH, D-52056 Aachen, FRG ${ }^{4}$

III. Physikalisches Institut, RWTH, D-52056 Aachen, FRG ${ }^{4}$

${ }^{\mathrm{b}}$ National Institute for High Energy Physics, NIKHEF, and University of Amsterdam, NL-1009 DB Amsterdam, The Netherlands ${ }^{\mathrm{c}}$ University of Michigan, Ann Arbor, MI 48109, USA

'Laboratoire d'Annecy-le-Vieux de Physique des Particules, LAPP,IN2P3-CNRS, BP 110, F-74941 Annecy-le-Vieux CEDEX, France

e Johns Hopkins University, Baltimore, MD 21218, USA

${ }^{\mathrm{f}}$ Institute of Physics, University of Basel, $\mathrm{CH}-4056$ Basel, Switzerland

${ }^{\mathrm{g}}$ Institute of High Energy Physics, IHEP, 100039 Beijing, China $^{5}$

${ }^{\mathrm{h}}$ Humboldt University, D-10099 Berlin, FRG ${ }^{4}$

${ }^{\mathrm{i}}$ University of Bologna and INFN-Sezione di Bologna, I-40126 Bologna, Italy

${ }^{\mathrm{j}}$ Tata Institute of Fundamental Research, Bombay 400005 , India

${ }^{\mathrm{k}}$ Boston University, Boston, MA 02215, USA

${ }^{1}$ Northeastern University, Boston, MA 02115, USA

${ }^{\mathrm{m}}$ Institute of Atomic Physics and University of Bucharest, R-76900 Bucharest, Romania

${ }^{n}$ Central Research Institute for Physics of the Hungarian Academy of Sciences, H-1525 Budapest 114, Hungary ${ }^{6}$

${ }^{\circ}$ Massachusetts Institute of Technology, Cambridge, MA 02139, USA

${ }^{\mathrm{p}}$ INFN Sezione di Firenze and University of Florence, I-50125 Florence, Italy

${ }^{\mathrm{q}}$ European Laboratory for Particle Physics, CERN, CH-1211 Geneva 23, Switzerland

${ }^{\mathrm{r}}$ World Laboratory, FBLJA Project, $\mathrm{CH}-1211$ Geneva 23, Switzerland

' University of Geneva, CH-1211 Geneva 4, Switzerland

${ }^{\mathrm{t}}$ Chinese University of Science and Technology, USTC, Hefei, Anhui 230 029, China ${ }^{5}$

" SEFT, Research Institute for High Energy Physics, P.O. Box 9, SF-00014 Helsinki, Finland

${ }^{\mathrm{V}}$ University of Lausanne, $\mathrm{CH}-1015$ Lausanne, Switzerland

${ }^{w}$ INFN-Sezione di Lecce and Universitá Degli Studi di Lecce, I-73100 Lecce, Italy

${ }^{x}$ Los Alamos National Laboratory, Los Alamos, NM 87544, USA

${ }^{y}$ Institut de Physique Nucléaire de Lyon, IN2P3-CNRS, Université Claude Bernard, F-69622 Villeurbanne, France

${ }^{\mathrm{z}}$ Centro de Investigaciones Energeticas, Medioambientales y Tecnologicas, CIEMAT, E-28040 Madrid, Spain ${ }^{7}$

aa INFN-Sezione di Milano, I-20133 Milan, Italy

${ }^{\mathrm{ab}}$ Institute of Theoretical and Experimental Physics, ITEP, Moscow, Russia

ac INFN-Sezione di Napoli and University of Naples, I-80125 Naples, Italy

ad Department of Natural Sciences, University of Cyprus, Nicosia, Cyprus

ae University of Nijmegen and NIKHEF, NL-6525 ED Nijmegen, The Netherlands

af Oak Ridge National Laboratory, Oak Ridge, TN 37831, USA 
ag California Institute of Technology, Pasadena, CA 91125, USA

ah INFN-Sezione di Perugia and Universitá Degli Studi di Perugia, I-06100 Perugia, Italy

ai Carnegie Mellon University, Pittsburgh, PA 15213, USA

aj Princeton University, Princeton, NJ 08544, USA

ak INFN-Sezione di Roma and University of Rome, "La Sapienza', I-00185 Rome, Italy

${ }^{\text {al }}$ Nuclear Physics Institute, St. Petersburg, Russia

am University and INFN, Salerno, I-84100 Salerno, Italy

an University of California, San Diego, CA 92093, USA

ao Dept. de Fisica de Particulas Elementales, Univ. de Santiago, E-15706 Santiago de Compostela, Spain

ap Bulgarian Academy of Sciences, Central Lab. of Mechatronics and Instrumentation, BU-1113 Sofia, Bulgaria

${ }^{\text {aq }}$ Center for High Energy Physics, Korea Adv. Inst. of Sciences and Technology, 305-701 Taejon, South Korea

ar University of Alabama, Tuscaloosa, AL 35486, USA

as Utrecht University and NIKHEF, NL-3584 CB Utrecht, The Netherlands

at Purdue University, West Lafayette, IN 47907, USA

${ }^{\text {au }}$ Paul Scherrer Institut, PSI, CH-5232 Villigen, Switzerland

${ }^{\text {av }}$ DESY-Institut fir Hochenergiephysik, D-15738 Zeuthen, FRG

${ }^{\text {aw }}$ Eidgenössische Technische Hochschule, ETH Zürich, CH-8093 Zürich, Switzerland

${ }^{a x}$ University of Hamburg, D-22761 Hamburg, FRG

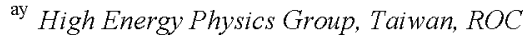

Received 18 December 1997

Editor: K. Winter

\begin{abstract}
Local multiplicity fluctuations in hadronic $Z$ decays are studied using the L3 detector at LEP. Bunching parameters are used for the first time in addition to the normalised factorial moment method. The bunching parameters directly demonstrate that the fluctuations in rapidity are multifractal. Monte Carlo models show agreement with the data, reproducing the trend, although not always the magnitude, of the factorial moments and bunching parameters. (c) 1998 Elsevier Science B.V. All rights reserved.
\end{abstract}

\section{Introduction}

Hadronic final states of $\mathrm{e}^{+} \mathrm{e}^{-}$collisions provide a favourable environment for QCD studies. Initially, the hadronic system is simply a quark-antiquark pair.

\footnotetext{
${ }^{1}$ Also supported by CONICET and Universidad Nacional de La Plata, CC 67, 1900 La Plata, Argentina.

${ }^{2}$ Also supported by Panjab University, Chandigarh-160014, India.

${ }^{3}$ Supported by Deutscher Akademischer Austauschdienst.

${ }^{4}$ Supported by the German Bundesministerium für Bildung, Wissenschaft, Forschung und Technologie.

${ }^{5}$ Supported by the National Natural Science Foundation of China.

${ }^{6}$ Supported by the Hungarian OTKA fund under contract numbers T14459, T19181 and T24011.

${ }^{7}$ Supported also by the Comisión Interministerial de Ciencia y Technología.
}

The energy of this pair is dissipated during a complex, non-linear QCD parton shower and non-perturbative hadronisation process. Monte Carlo (MC) programs incorporating the QCD parton shower and phenomenological models of hadronisation and resonance decay have been successful in describing global features.

In this paper we use high statistics data from the L3 experiment at LEP to study fluctuations in the charged particle multiplicity distribution in small regions of phase space, as a function of the size of the region. If particles were independently produced, the local multiplicity distribution would be a Poissonian. A deviation of this distribution from a Poissonian measures dynamical local multiplicity fluctuations, which are a consequence of short-range correlations between final-state particles. Parton showers, fragmentation, resonance decays and Bose-Einstein interference can all contribute to these correlations. 
Fluctuations are often studied using the normalised factorial moments (NFMs) of the local multiplicity distribution, $P_{n}(\delta)$, which is the probability to find $n$ particles inside a phase space bin of size $\delta$ [1]. The NFM of order $q, F_{q}(\delta)$, is defined by

$$
\begin{aligned}
& F_{q}(\delta)=\frac{\left\langle n^{[q]}\right\rangle}{\langle n\rangle^{q}}, \\
& \left\langle n^{[q]}\right\rangle=\sum_{n=q}^{\infty} n^{[q]} P_{n}(\delta), \\
& n^{[q]}=n(n-1) \ldots(n-q+1) .
\end{aligned}
$$

If $P_{n}(\delta)$ is a Poisson distribution, $F_{q}(\delta)=1$ for all $q$. If there are fluctuations, $F_{q}(\delta)$ deviates from unity. Further, if the fluctuations are self-similar, i.e., $F_{q}(\lambda \delta)=\lambda^{-\phi_{q}} F_{q}(\delta)$, then a power-like increase emerges with decreasing $\delta, F_{q}(\delta) \propto \delta^{-\phi_{q}}$, where the intermittancy indices $\phi_{q}=(q-1) d_{q}$, and $d_{q}$ are the anomalous fractal dimensions. Local fluctuations are classified as monofractal $\left(d_{q}\right.$ is independent of $q$ ) or multifractal ( $d_{q}$ is a function of $q$ ). (See recent reviews [2].)

Local fluctuations in $\mathrm{e}^{+} \mathrm{e}^{-}$processes have been studied in several experiments $[3-5,7,8,6,9,11,10]$. The data do indeed exhibit an approximate power-like rise of the NFMs with decreasing $\delta$, especially when evaluated in two- and three-dimensional phase space variables. All four LEP experiments have found that current MC models can, in general, describe the NFMs, even without additional tuning. Exceptions have, however, been found in rapidity defined with respect to the sphericity axis by OPAL [7] and by DELPHI [9] (for restricted charge-multiplicity and $p_{\mathrm{T}}$ regions).

Recently, it has been realized that the factorial moment method poorly reflects the information on local fluctuations, since the NFM of order $q$ contains a contamination from lower-order correlation functions [2]. As a result, the nature of the fluctuations is difficult to determine from the behaviour of the NFMs. Factorial moments also suffer from a statistical bias due to the finite size of the event sample. This is because measurements of the NFMs are dominated by the first few terms of expression (2). In most cases this leads to a significant underestimate of the measured NFMs with respect to their true values [12].
An alternative to the NFMs is provided by bunching parameters (BPs), $\eta_{q}(\delta),[13,14]$ which are defined by

$$
\eta_{q}(\delta)=\frac{q}{q-1} \frac{P_{q}(\delta) P_{q-2}(\delta)}{P_{q-1}^{2}(\delta)}, \quad q=2,3, \ldots
$$

They are more sensitive than the NFMs to the variation in the shape of $P_{n}(\delta)$ with decreasing $\delta$. In the case of self-similar fluctuations, one expects $\eta_{2}(\delta)$ $\alpha \delta^{-d_{2}}$. For multifractal local fluctuations, the $\eta_{q}(\delta)$ are $\delta$-dependent functions for all $q \geq 3$, while for monofractal behaviour $\eta_{q}(\delta)$ is independent of $\delta$ for $q \geq 3$ [13]. For independent particle production, the BPs are $\delta$-independent constants (for a Poisson distribution, $\left.\eta_{q}(\delta)=1\right)$, for all $q$.

From an experimental point of view, the BPs have several advantages [14]: (1) They are less severely affected by statistical bias than the NFMs, since the BP of order $q$ depends only on the behaviour of the multiplicity distribution near multiplicity $n=q-1$; (2) for the calculation of the BP of order $q$, one only needs to be able to resolve $q$ particles in a bin, rather than all particles as for the NFMs; and (3) many systematic errors cancel in the ratio of probabilities.

When defined in this way, the NFMs and BPs both require dividing phase space into a number of bins. This has the disadvantage of losing information on local fluctuations in particle density ("spikes") that are divided by bin boundaries. To remedy this problem with the NFMs, and in addition to increase the effective statistics, normalized density strip integrals have been proposed [15]. Analogously, a new type of bunching parameter has been suggested [14], which can be used to study the fluctuation of the number of spikes (defined in Section 2) per event Generalized integral bunching parameters (GBPs) are defined by

$\chi_{q}(\epsilon)=\frac{q}{q-1} \frac{\Pi_{q}(\epsilon) \Pi_{q-2}(\epsilon)}{\Pi_{q-1}^{2}(\epsilon)}$,

where $\Pi_{q}(\epsilon)$ is the probability of an event to have $q$ spikes of size less than $\epsilon$, irrespective of how many particles are inside each spike. For purely independent particle production, with the multiplicity distribution characterised by a Poissonian, $\chi_{q}(\epsilon)=1$ for all $q$. 
In this paper we study fluctuations in rapidity, defined with respect to the thrust axis, using factorial moments and, for the first time, bunching parameters. We also study fluctuations in the four-momentum difference using generalized bunching parameters.

\section{Methods}

In order to improve the accuracy we use the bin-averaged "horizontal" NFMs [1] and BPs $[13,14]$ : The NFM of order $q$ is calculated using the standard definition:

$F_{q}(M)=\frac{1}{M} \sum_{m=1}^{M} \frac{\left\langle n_{m}^{[q]}\right\rangle}{\langle\bar{n}\rangle^{q}}$,

$n_{m}^{[q]}=n_{m}\left(n_{m}-1\right) \ldots\left(n_{m}-q+1\right)$,

where $n_{m}$ is the number of particles in bin $m$, $\langle\bar{n}\rangle=\bar{N} / M, \bar{N}$ is the average multiplicity for full phase space, $M=\Delta / \delta$ is the total number of bins, and $\Delta$ represents the full phase space volume. The "horizontal" BP of order $q$ is calculated using

$\eta_{q}(M)=\frac{q}{q-1} \frac{\bar{N}_{q}(M) \bar{N}_{q-2}(M)}{\bar{N}_{q-1}^{2}(M)}$,

$\bar{N}_{q}(M)=\frac{1}{M} \sum_{m=1}^{M} N_{q}(m, \delta)$,

where $N_{q}(m, \delta)$ is the number of events having $q$ particles in bin $m$ and $M$ has the same meaning as for the NFMs.

Note that bin-averaging, as used in the above definitions, is only justified for a flat single-particle density distribution. To be able to study non-flat distributions, we transform the original phase space variable to one in which the underlying density is uniform [16].

For the generalized bunching parameters we need to define the number of spikes of size less than $\epsilon$. To do so we need both a measure of the size of a spike and a method of assigning particles to spikes. For the spike size, $\sigma$, we use the so-called GrassbergerHentschel-Procaccia counting topology [17] for which $\sigma$ is the maximum of all pairwise distances between particles in the spike. As distance between particles $i$ and $j$ we use the squared four-momentum difference $Q_{i j}^{2}=-\left(p_{i}-p_{j}\right)^{2}$. Spikes are then defined in the following way: Consider all possible combinations of two or more particles. For each combination $\sigma$ is determined. If $\sigma$ is larger than some maximum, $\epsilon$, the combination is discarded. Of the remaining combinations, those completely contained in another (larger) combination are also discarded. Combinations left after this procedure are called spikes (of size less than $\epsilon$ ). Note that while the assignment of particles to spikes is not unique (the same particle can be in more than one spike), the number of spikes is unambiguously defined.

The GBPs are then given by

$\chi_{q}\left(Q^{2}\right)=\frac{q}{q-1} \frac{S_{q}\left(Q^{2}\right) S_{q-2}\left(Q^{2}\right)}{S_{q-1}^{2}\left(Q^{2}\right)}$,

where $S_{q}\left(Q^{2}\right)$ is the number of events having $q$ spikes of size less than $Q^{2}$.

\section{Data samples and analysis procedures}

We use data, corresponding to an integrated luminosity of $52 \mathrm{pb}^{-1}$, collected at a centre of mass energy of $\sqrt{s} \simeq 91.2 \mathrm{GeV}$ during the 1994 LEP running period. Hadronic events are selected using (1) energy deposits in the electromagnetic and hadronic calorimeters, and (2) momenta measured in the Time Expansion Chamber (TEC) and the Silicon Microvertex Detector (SMD). The L3 detector is described in detail in Ref. [18].

First, a loose calorimeter-based selection is performed in order to reject non-hadronic background. Using clusters with energy larger than $100 \mathrm{MeV}$, we require

$0.6<\frac{E^{\mathrm{C}}}{\sqrt{s}}<1.4, \quad \frac{E_{\perp}}{E^{\mathrm{C}}}<0.4, \quad \frac{E_{\|}}{E^{\mathrm{C}}}<0.4$,

$13<N_{\mathrm{cl}}<75$,

where $E^{\mathrm{C}}$ is the total energy observed in the calorimeters, $E_{\perp}\left(E_{\|}\right)$is the energy imbalance in the plane perpendicular (parallel) to the beam direction, and $N_{\mathrm{cl}}$ is the number of calorimeter clusters. To ensure that the event is contained in the barrel region of the calorimeters we require $\left|\cos \theta_{\text {thr }}\right|<0.74$, where $\theta_{\mathrm{thr}}$ is the polar angle of the event thrust axis. 
To obtain a sample with well-measured charged tracks, a further selection is performed using only tracks which have passed certain quality cuts. The distance of closest approach (projected onto the transverse plane) of a track to the nominal interaction vertex is required to be less than $5 \mathrm{~mm}$ and its transverse momentum must be larger than $100 \mathrm{MeV}$. To ensure that the event lies within the full acceptance of the TEC and SMD, the direction of the thrust axis, as determined from the charged tracks, must satisfy $\left|\cos \theta_{\text {thr }}\right|<0.7$. Events are then selected using criteria similar to the above calorimeter-based selection, but using tracks:

$$
\begin{aligned}
& \frac{\sum_{i} p_{i}}{\sqrt{s}}>0.15, \quad \frac{\left|\sum_{i} p_{\| i}\right|}{\sum_{i} p_{i}}<0.75, \\
& \frac{\left|\sum_{i} \boldsymbol{p}_{\perp i}\right|}{\sum_{i} p_{i}}<0.75, \quad N_{\mathrm{ch}}>4,
\end{aligned}
$$

where $p_{i}$ is the momentum of particle $i$ and the sum runs over all tracks of an event, and where $N_{\text {ch }}$ is the number of charged tracks. The resulting sample contains about 1 million events.

The experimental distributions are corrected for selection and acceptance losses using two samples, "generator level" and "detector level" of $\mathrm{e}^{+} \mathrm{e}^{-} \rightarrow$ hadrons MC events generated with JETSET 7.4 PS [19] including initial-state photon radiation. At the generator level particles with lifetime $c \tau>1 \mathrm{~cm}$ are assumed stable. The detector level sample has passed a full detector simulation [20] including time-dependent variations of the detector response based on continuous detector monitoring and calibration. It has been reconstructed with the same program as the data and passed through the same selection procedure.

In this paper we study fluctuations in the rapidity with respect to the thrust axis, $y$, and the square of the pairwise four-momentum difference, $Q^{2}$. Both for the calculation of $Q^{2}$ and for the grouping of tracks into small bins of rapidity, the resolution of the angle between pairs of tracks is of crucial importance. For this reason we impose additional stringent quality cuts on track reconstruction, which results in rejection of $39 \%$ of the tracks. With this selection we achieve very good agreement between data and simulation for the distributions of the difference in angle between pairs of tracks for both the azimuthal angle about, and the polar angle with respect to, the beam, as is shown in Fig. 1a and $1 \mathrm{~b}$, respectively.

The uncorrected distributions of $y$, and $Q^{2}$, are compared to the detector level $\mathrm{MC}$ distributions in Figs. $1 \mathrm{c}$ and $1 \mathrm{~d}$, respectively. There is reasonable agreement, which indicates the quality of both the detector simulation and the JETSET predictions. It should be noted that these distributions have not been used in the tuning of JETSET's parameters.

The distributions of NFMs and BPs are corrected bin-by-bin for detector effects. The corrected value
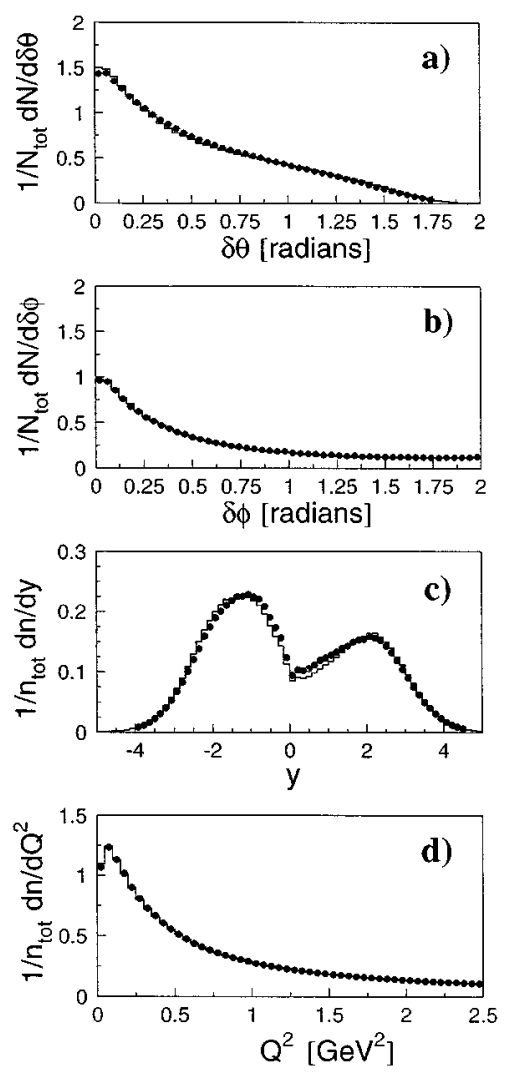

Fig. I. Distributions of (a) the difference in polar angle of pairs of tracks, $\delta \theta$, (b) the difference in azimuthal angle of pairs of tracks, $\delta \phi$, (c) the single-particle rapidity with respect to the thrust axis, $y$, and (d) the inclusive four-momentum difference squared, $Q^{2}$, for uncorrected data (points) compared to the predictions of JETSET after detector simulation (histogram). 
in a bin is found by multiplying the value calculated directly from the data by a correction factor given by the ratio $C_{q}=M_{q}^{\text {gen }} / M_{q}^{\text {det }}$, where $M_{q}^{\text {gen }}$ and $M_{q}^{\text {det }}$ are the corresponding NFM or BP calculated from the generator- and detector-level MC samples, respectively. These corrections, which tend to be larger for smaller bin size, are in no case larger than about $5 \%$.

To reduce possible systematic bias, the minimum bin size is chosen comparable to the experimental resolution, which was estimated $[21,22]$ by $\mathrm{MC}$ simulation. In the case of $Q^{2}$, the smallest bin size is large enough that the measurements are not strongly affected by Dalitz decays $\left(\pi^{0} \rightarrow \mathrm{e}^{+} \mathrm{e}^{-} \gamma\right)$ or by photon conversions.

The error bars on the results include contributions from both statistical and systematic errors on the raw quantities and on the correction factors. The statistical errors on the $F_{q}(M)$ and $\eta_{q}(M)$ are derived from the covariance matrix of the NFMs and BPs. The statistical errors for the GBPs are derived according to the expression obtained in Ref. [14]. Systematic errors on the raw quantities have been estimated by varying the event- and track-selection criteria. As systematic error on $C_{q}$, we take half of the difference between the correction factors determined using JETSET and those using HERWIG.

The predictions of the JETSET 7.4 PS [19], ARIADNE 4.08 [23] and HERWIG 5.9 [24] models, all of which have been tuned to reproduce global event-shape and single-particle inclusive distributions $[10,25]$, are compared to the data. The BoseEinstein effect is a potential source of particle correlations. JETSET and ARIADNE include the same modelling of this effect. ${ }^{8}$ HERWIG does not incorporate a Bose-Einstein model. We also compare the data with predictions of JETSET without Bose-Einstein interference. ${ }^{9}$ The errors on the JETSET predictions include both statistical and systematic errors. These systematic errors were estimated by varying, by one standard deviation, the following JETSET parameters tuned in Ref. [10,25]: the Lund fragmen-

\footnotetext{
${ }^{8}$ The Bose-Einstein model used is the luboei model of JETSET.

${ }^{9}$ The parameters of JETSET were retuned with Bose-Einstein interference disabled. This resulted in changes of $\operatorname{PARJ}(21)$, PARJ(42), and PARJ(81) from 0.411, 0.886, and 0.311 to 0.343 , 1.1 , and 0.312 , respectively.
}

tation function parameter $b$, the width of the Gaussian $p_{x}$ and $p_{y}$ hadronic transverse momentum distribution, and the value of $\Lambda$ used for $\alpha_{\mathrm{s}}$ in parton showers. Systematic errors on the other MC predictions are similarly determined. The errors on the ARIADNE predictions are comparable to those on JETSET, while those of HERWIG are about 50\% larger. The errors on the MC results are dominated by the systematic errors.

\section{Results}

\subsection{Fluctuations in rapidity, $y$}

To study fluctuations inside jets, we first determine the thrust axis and analyse the NFMs and BPs in the full rapidity range $|y| \leq 5$. Since the singleparticle rapidity distribution is non-uniform, we first transform $y$ to a uniformly distributed variable [16].

The horizontally normalised NFMs are shown in Fig. 2 as a function of the number of bins, $M$, in the

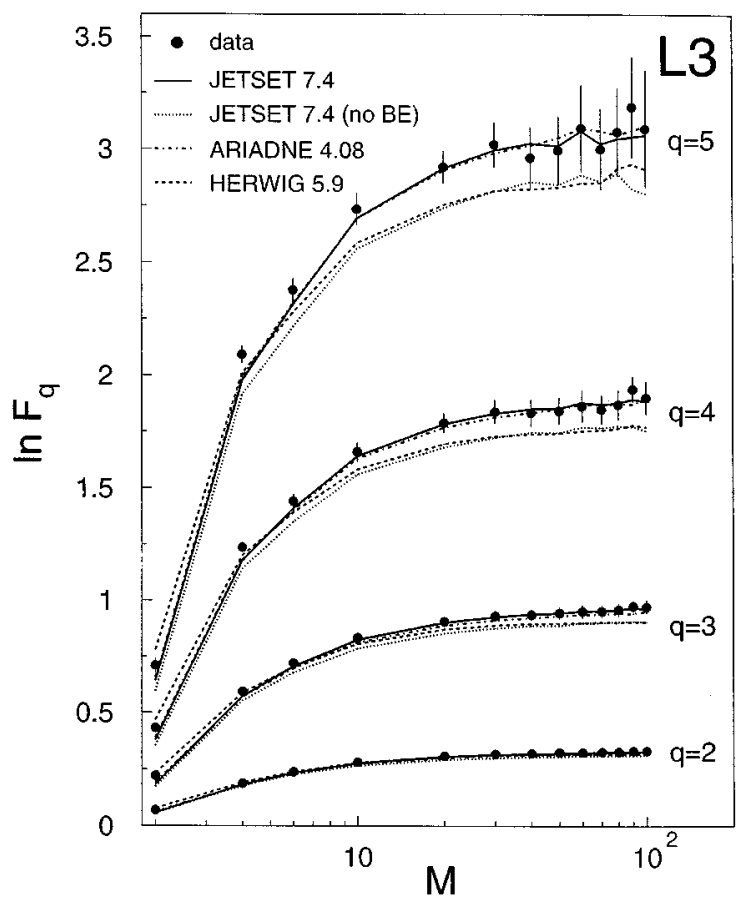

Fig. 2. NFMs as a function of the number of bins, $M$, in the transformed rapidity defined with respect to the thrust axis. In this and the following figures the shaded areas represent the errors on the JETSET predictions. The errors on the ARIADNE predictions are comparable whereas those on the HERWIG predictions are about $50 \%$ larger. 


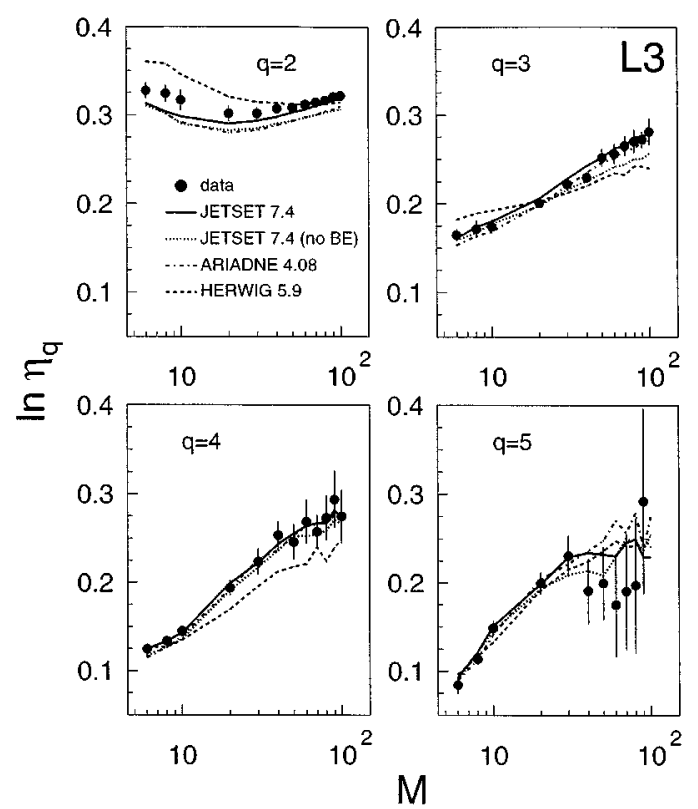

Fig. 3. BPs as a function of the number of bins, $M$, in the transformed rapidity defined with respect to the thrust axis.

transformed rapidity. They rise with increasing $M$ and then saturate. All of the MCs are in reasonable agreement with the data. It is also seen that the Bose-Einstein effect in JETSET raises the values of the NFMs.

Fig. 3 shows the results for the horizontally normalised BPs. All higher-order $(q>2)$ BPs show an approximate power-law increase with increasing $M$. The MCs also show a power-law behaviour, although some differences in slope are apparent. That these BPs vary with $M$ is a direct indication that the fluctuations in $y$ are multifractal ${ }^{10}[13]$, as is expected in QCD [26-28].

The second-order BP decreases with increasing $M$ up to $M \approx 20$, which is found to correspond to the value of $M$ at which the maximum of the multiplicity distribution $P_{n}(\delta)$ first occurs at $n=0$. The large errors on the data for $M \leqq 30$ arise mainly

\footnotetext{
${ }^{10}$ This conclusion is possible without measuring the intermittency indices $\phi_{q}$. In contrast, to reveal multifractality with NFMs one must first fit the NFMs by a power law. Because of the saturation of the $F_{q}(M)$ observed in Fig. 2, this procedure is fraught with ambiguity.
}

from the systematic error assigned from the difference between correction factors determined using JETSET and HERWIG. The data are shown using the JETSET correction factors.

The second-order BP is related to the width of the multiplicity distribution $[13,14]$. Hence, HERWIG's overestimation of $\eta_{2}$ means that HERWIG's local multiplicity distributions are too broad. This agrees with ALEPH's conclusion from a direct measurement of the dispersion for various (large) intervals of rapidity [29], but emphasizes the contribution of the low values of $n$ in this discrepancy.

To study the second-order BP in more detail, we split $\eta_{2}$ into two components:

$\boldsymbol{\eta}_{2}(M)=\boldsymbol{\eta}_{2}^{( \pm \pm)}(M)+\boldsymbol{\eta}_{2}^{(+-)}(M)$.

The definition of $\eta_{2}^{( \pm \pm)}(M)$ is as in Eq. (6), but with $N_{2}(m, \delta)$ replaced by $N_{2}^{( \pm \pm)}(m, \delta)$, the number of events having two like-charged particles inside bin $m$ of size $\delta$. Analogously, $\eta_{2}^{(+-)}(M)$ is constructed from the number of events having two oppositely charged particles in the bin. Note that for combinatorial reasons, $\eta_{2}^{( \pm \pm)}(M)<\eta_{2}^{(+-)}(M)$. However, both would be independent of $M$ in the case of independent production.

Fig. 4 shows that $\eta_{2}^{( \pm \pm)}(M)$ and $\eta_{2}^{(+-)}(M)$ behave differently. While $\eta_{2}^{( \pm \pm)}(M)$ shows the expected rise (and saturation of the data at large $M$ ), $\eta_{2}^{(+-)}(M)$ shows a decrease at low $M$.

The anti-bunching tendency (decrease of $\eta_{2}$ with increasing $M$ ) seen for unlike-charged particles for $M \lesssim 20$ is also seen in all MCs. Resonance decays

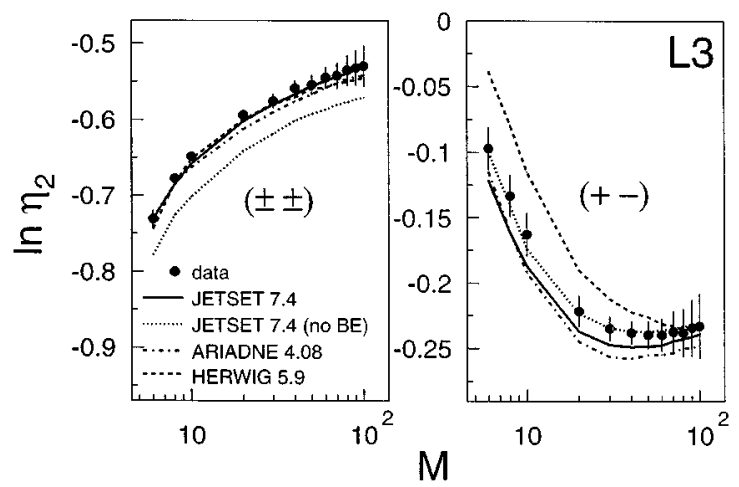

Fig. 4. The second-order BP as a function of the number of bins, $M$, in the transformed rapidity defined with respect to the thrust axis for like-charged and unlike-charged particle combinations. 
are a likely explanation of this effect. Their decay particles tend to be of opposite charge and are typically separated in rapidity by $\delta y \sim 0.5-1.0$. As a result, a rapidity separation of this order of magnitude is more frequent between unlike-charged particles than between like-charged and $\eta_{2}^{(+-)}(M)$ is much larger than $\eta_{2}^{( \pm \pm)}(M)$ at small $M$ (large $\delta y$ ). However, this difference decreases rapidly with decreasing $\delta y$ until about $M=20$, which corresponds to $\delta y=0.5$.

For like-signed combinations the MCs all show good agreement with the data. However, for unlikecharged combinations HERWIG overestimates the data, while the other MCs agree reasonably well. The errors on the JETSET predictions are dominantly systematic. They are mainly due to the uncertainty on the parameter responsible for the width of the Gaussian hadronic transverse momentum distribution in the Lund model. This shows the importance of fragmentation for the bunching parameters. For $\eta_{2}^{(+-)}$, as for $\eta_{2}$, the error at small $M$ comes mainly from differences in the correction factors; other errors are comparable to the errors on the corresponding points for $\eta_{2}^{( \pm \pm)}$. The effect of BoseEinstein interference in JETSET is to increase the value of $\eta_{2}^{( \pm \pm)}$and to decrease that of $\eta_{2}^{(+-)}$.

\subsection{Fluctuations in four-momentum difference, $Q^{2}$}

The behaviour of the GBPs for the invariant two-particle squared four-momentum difference, $\chi_{q}\left(Q^{2}\right)$, is shown in Fig. 5 as a function of $-\ln Q^{2}$. All GBPs, for both data and Monte Carlo, rise similarly with increasing $-\ln Q^{2}$ (decreasing $Q^{2}$ ). This corresponds to a bunching effect for all orders, similar to the behaviour of fluctuations in $y$. The MC models show a similar trend with $-\ln Q^{2}$ but tend to underestimate the values of $\chi_{q}\left(Q^{2}\right)$; HERWIG agrees best with the data.

Given the difference in behaviour observed in the previous section between $\eta_{2}^{(+-)}$and $\eta_{2}^{( \pm \pm)}$, we now define second-order GBPs for multiparticle spikes consisting entirely of particles of the same charge, $\chi_{2}^{(s c)}$, and for spikes containing particles of different charge, $\chi_{2}^{(d c)}$. These GBPs are defined as in Eqs. (4) and (7) except that $q$ now refers to the number of $s C$ and $d c$ spikes, respectively. We plot in Fig. 6 the behaviour of $\chi_{2}^{s c}$ and $\chi_{2}^{d c}$. A difference is observed

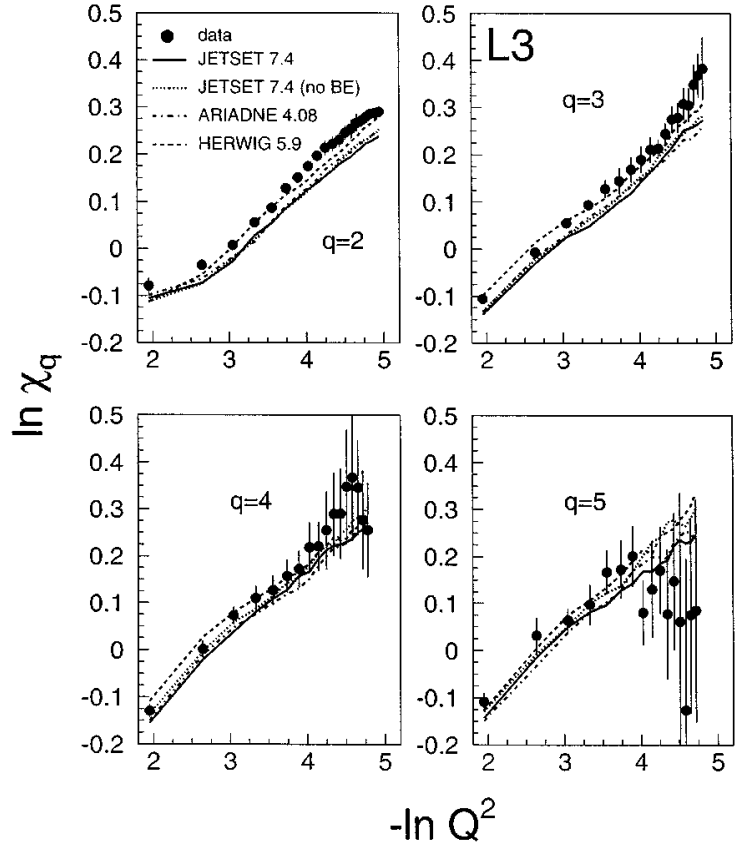

Fig. 5. GBPs as a function of the squared four-momentum difference $Q^{2}$ in $\mathrm{GeV}^{2}$ between two charged particles.

between these two quantities. For sc spikes a strong bunching effect $\left(\chi_{2}^{(s c)}\left(Q^{2}\right)>1\right)$ is seen at large $-\ln Q^{2}$. This is well reproduced by HERWIG. In the case of $d c$ spikes, the bunching is smaller and tends to saturate at large $-\ln Q^{2}$.

In contrast to the BPs of the previous section, resonances have little effect on the GBPs. This is

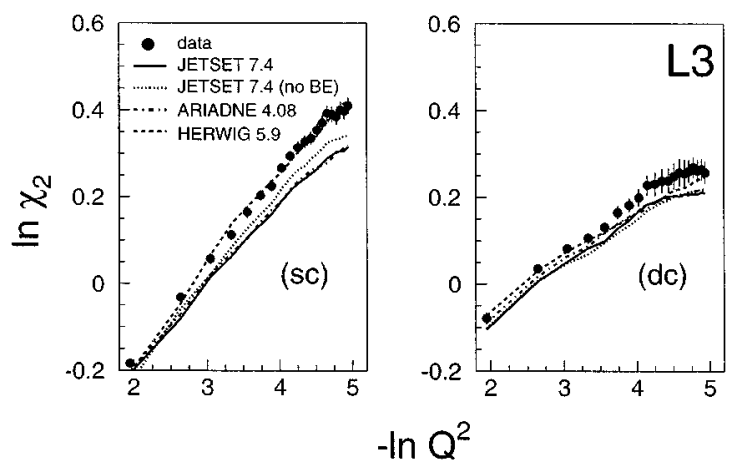

Fig. 6. The second-order GBP for spikes of same-charged particles (sc) and spikes of differently charged particles $(d c)$ as a function of the squared four-momentum difference $Q^{2}$ in $\mathrm{GeV}^{2}$ between two charged particles. 
because the most copiously produced resonances decay to two particles with a $Q^{2}$ so large that the particles are necessarily in different spikes for the spike sizes which we consider $\left(-\ln Q^{2} \geq 2\right)$. The spike multiplicity distribution is therefore not strongly affected.

It is interesting to observe that JETSET's treatment of BE correlations decreases the values of $\chi_{2}$ for $S C$ spikes, the opposite of the behaviour for $\eta_{2}^{( \pm \pm)}$. This comes about because JETSET's modelling of Bose-Einstein interference increases the number of sc spikes. This leads to higher values for $\eta_{2}^{( \pm \pm)}$. However, $\chi_{2}$ is mainly influenced by the shape of the spike-multiplicity distribution rather than by the average spike multiplicity.

\section{Conclusions}

Local charged particle multiplicity fluctuations in rapidity with respect to the thrust axis have been studied using factorial moments and, for the first time, bunching parameters, which are sensitive to further details. Also, fluctuations of spikes have been studied using generalized bunching parameters defined in terms of the four-momentum difference, $Q^{2}$. Bunching parameters directly demonstrate a multifractal behaviour of the fluctuations in rapidity, as is expected from QCD.

Monte Carlo models, which have been tuned to reproduce global event-shape distributions and single-particle inclusive distributions provide a reasonable description of fluctuations in these variables. They reproduce the trend, although not always the magnitude, of the normalized factorial moments, bunching parameters and generalized integral bunching parameters. It thus appears that the ingredients of these MC models (coherent parton shower, string or cluster fragmentation, and resonance decays) are sufficient to explain the fluctuations observed in the data.

\section{Acknowledgements}

We wish to express our gratitude to the CERN accelerator divisions for the excellent performance of the LEP machine. We acknowledge the effort of all engineers and technicians who have participated in the construction and maintenance of this experiment. We thank V. I. Kuvshinov, J.-L. Meunier and R. Peschanski for useful discussions and comments.

\section{References}

[1] A. Białas, R. Peschanski, Nucl. Phys. B 273 (1986) 703; Nucl. Phys. B 308 (1988) 857.

[2] P. Bożek, M. Płoszajczak, R. Botet, Phys. Rep. 252 (1995) 101; E.A. De Wolf, I.M. Dremin, W. Kittel, Phys. Rep. 270 (1996) 1.

[3] TASSO Collaboration, W. Braunschweig et al., Phys. Lett. B 231 (1989) 548.

[4] K. Sugano, HRS Collaboration, in: F. Cooper et al. (Eds.), Proc. Santa Fe Workshop Intermittency in High-Energy Collisions, World Scientific, Singapore, 1991, p. 1.

[5] CELLO Collaboration, H.J. Behrend et al., Phys. Lett. B 256 (1991) 97.

[6] DELPHI Collaboration, P. Abreu et al., Phys. Lett. B 247 (1990) 137; A. De Angelis, Mod. Phys. Lett. A 5 (1990) 2395.

[7] OPAL Collaboration, M.Z. Akrawy et al., Phys. Lett. B 262 (1991) 351.

[8] ALEPH Collaboration, D. Decamp et al., Z. Phys. C 53 (1992) 21.

[9] DELPHI Collaboration, P. Abreu et al., Nucl. Phys. B 386 (1992) 471.

[10] L3 Collaboration, B. Adeva et al., Z. Phys. C 55 (1992) 39.

[11] F. Mandl, B. Buschbeck, DELPHI Collaboration, in: A Giovannini et al. (Eds.), Proc. 24 Int. Symp. on Multiparticle Dynamics, Vietri sul Mare, Italy, 1994, World Scientific, Singapore, 1995, p. 52; B. Buschbeck, F. Mandl, ICHEP'96 Ref. pa01-028; B. Buschbeck, P. Lipa, F. Mandl, DELPHI Collaboration, in: R.C. Hwa et al. (Eds.) Proc. 7th Int. Workshop on Multiparticle Production Correlations and Fluctuations, Nijmegen, The Netherlands, 1996, World Scientific, Singapore, 1997, p. 175.

[12] E.M. Friedlander, Mod. Phys. Lett. A 4 (1989) 2457; W. Kittel, in: F. Cooper et al. (Eds.), Proc. Santa Fe Workshop Intermittency in High-Energy Collisions, World Scientific, Singapore, 1991, p. 83; P. Lipa et al., Z. Phys. C 54 (1992) 115.

[13] S.V. Chekanov, V.I. Kuvshinov, Acta Phys. Polon. B 25 (1994) 1189; S.V. Chekanov, W. Kittel, V.I. Kuvshinov, Acta Phys. Polon. B 27 (1996) 1739.

[14] S.V. Chekanov, W. Kittel, V.I. Kuvshinov, Z. Phys. C 74 (1997) 517.

[15] P. Lipa et al., Phys. Lett. B 285 (1992) 300.

[16] A. Białas, M. Gazdzicki, Phys. Lett. B 252 (1990) 483; W. Ochs, Z. Phys. C 50 (1991) 339.

[17] P. Grassberger, Phys. Lett. A 97 (1983) 227; H. Hentschel, I. Procaccia, Physica D 8 (1983) 43. 
[18] L3 Collaboration, B. Adeva et al., Nucl. Instrum. Methods A 289 (1990) 35; J.A. Bakken et al., Nucl. Instrum. Methods A 275 (1989) 81; O. Adriani et al., Nucl. Instrum. Methods A 302 (1991) 53; B. Adeva et al., Nucl. Instrum. Methods A 323 (1992) 109; K. Deiters et al., Nucl. Instrum. Methods A 323 (1992) 162; B. Acciarri et al., Nucl. Instrum. Methods A 351 (1994) 300.

[19] T. Sjöstrand, Comput. Phys. Commun. 82 (1994) 74.

[20] The L3 detector simulation is based on GEANT3, see R. Brun et al., CERN report CERN DD/EE/84-1 (Revised), 1987, and uses GHEISHAto simulate hadronic interactions, see H. Fesefeldt, RWTH Aachen report PITHA 85/02, 1985.

[21] S.V. Chekanov, Local Multiplicity Fluctuations and Intermittent Structure Inside Jets, Ph.D. thesis, Univ. of Nijmegen, The Netherlands, 1997, ISBN 90-9010518-2.

[22] A.A. Syed, Particle Correlations in Hadronic Decays of the $Z$
Boson, Ph.D. thesis, Univ. of Nijmegen, The Netherlands, 1994, ISBN 90-9007038-9.

[23] L. Lönnblad, Comput. Phys. Commun. 71 (1992) 15.

[24] G. Marchesini, B.R. Webber, Nucl. Phys. B 310 (1988) 461; G. Marchesini et al., Comput. Phys. Commun. 67 (1992) 465.

[25] S. Banerjee, S. Banerjee, L3 Note 1978, 1996; I.G. Knowles, T. Sjöstrand (conveners), QCD Event Generators, Physics at LEP2, CERN-96-01, vol. 2, 1996, p. 103.

[26] W. Ochs, J. Wosiek, Phys. Lett. B 289 (1992) 159; B 304 (1993) 144; Z. Phys. C 68 (1995) 269.

[27] Yu. Dokshitzer, I.M. Dremin, Nucl. Phys. B 402 (1993) 139.

[28] Ph. Brax, J.-L. Meunier, R. Peschanski, Z. Phys. C 62 (1994) 649.

[29] ALEPH Collaboration, D. Buskulic et al., Z. Phys. C 69 (1995) 15. 